\section{DOSSIÊE}

Recebido em: 29/02/2014

Aceito em: 04/08/2014

\title{
Organización reticular de la memoria semántica. Natural Finder y Definition Finder, dos métodos informatizados para recuperar conocimiento Reticular organization of semantic memory. Natural and Finder Definition finder, two computerized methods for retrieving knowledge
}

\author{
Jorge VIVAS 1 \\ Francisco LIZARRALDE ${ }^{2}$ \\ Constanza HUAPAYA ${ }^{3}$ \\ Leticia VIVAS ${ }^{4}$ \\ Ana COMESAÑA ${ }^{5}$
}

\section{RESUMEN}

Dos líneas de teorías estudian la organización del conocimiento conceptual: las holísticas y las de rasgos. El objetivo de este trabajo es presentar dos métodos informatizados de captura y análisis de redes semánticas, el Natural Finder y el Definition Finder, que responden a estos modelos respectivamente. El primero es un procedimiento recursivo para capturar a partir de un concepto disparador la red semántica natural de una persona en torno a dicho concepto; el segundo permite obtener el conjunto de atributos ponderados que mejor definen a un concepto para un colectivo social. Se presentan ejemplos de su aplicación.

PALABRAS CLAVE: Red semántica. Métodos de exploración. Natural finder. Definition finder.

\begin{abstract}
Two types of theories address the organization of conceptual knowledge: holistic and featurebased. The aim of this paper is to present two computerized methods to capture and analyze semantic networks, the Natural Finder and the Definition Finder, which belong to these models respectively. The first is a recursive procedure to capture the natural semantic network of a person around a target concept; the second shows as a result the set of weighted defining features for certain concept given by a social group. Examples of its application are presented.
\end{abstract}

KEYWORDS: Semantic network. Exploration methods.; natural finder.; definition finder. Modelos de memoria semántica.

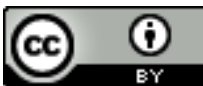

Esta obra está licenciada sob uma Licença Creative Commons.

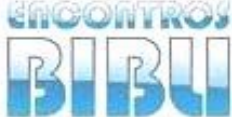

v. 19, n. 40,2014 p. $235-252$

ISSN 1518-2924
${ }^{1}$ CIMEPB, Facultad de Psicología, UNMDP - jvivas@mdp.edu.ar

${ }^{2}$ IAAI, Facultad de Ingeniería, UNMDP - flizarra@fi.mdp.edu.ar

${ }^{3}$ IAAI, Facultad de Ingeniería, UNMDP - huapaya@fi.mdp.edu.ar

${ }^{4}$ CIMEPB, CONICET, Facultad de Psicología, UNMDP - lvivas@mdp.edu.ar

${ }^{5}$ CIMEPB, CONICET, Facultad de Psicología, UNMDP - acomesan@mdp.edu.ar 


\section{INTRODUCCIÓN}

Existe cierto consenso acerca de que la información contenida en la memoria semántica se encuentra representada en forma de red (MCNAMARA; HOLBROOK, 2003). Tanto las teorías holísticas, que consideran que los conceptos están representados de manera unitaria mediante nodos (COLLINS; LOFTUS, 1975; DE ALMEIDA, 1999), como las teorías de rasgos (SMITH; MEDIN, 1981; CREE; MCRAE, 2003) que consideran que están representadas mediante conjuntos de rasgos o atributos, son compatibles con la idea de que la información semántica está representada en forma de una red donde los conceptos más semejantes en cuanto a significado se encuentran más próximos y los más disimiles se encuentran más lejanos. Para el primer tipo de modelos, cuando dos conceptos tienen significados similares habría una proximidad de los nodos que representan a cada concepto y una mayor fortaleza del vínculo que los une (COLLINS; LOFTUS, 1975). Para el segundo tipo de teoría los conceptos con significado similar estarían representados mediante patrones de atributos superpuestos dado que, tendrían gran cantidad de rasgos en común (MOSS; TYLER; TAYLOR, 2007).

Desde ambas teorías ha surgido el interés en conocer cómo está representado el significado de las palabras. Sin embargo, los métodos experimentales utilizados difieren en función de los supuestos de cada modelo. Para las teorías holísticas el significado de la palabra está representado por nodos lexicales, es decir que hay una parte de la estructura conceptual que representa al concepto holístico y se corresponde con una palabra (DE ALMEIDA, 1999). De este modo, la representación mental para cada cosa, evento o propiedad que pueda ser lexicalizada en el lenguaje es representada de manera unitaria y abstracta en el sistema conceptual del hablante (VIGLIOCCO; VINSON, 2007). En consecuencia, un método de exploración de la red semántica debería permitir conocer cuáles son las palabras que están vinculadas entre sí y cuál es la fuerza de su vinculación. 
Por su parte, para los modelos de rasgos el significado de la palabra no es unitario sino que puede ser descompuesto y es concebido como una combinación de rasgos o atributos definidores (CREE; MCRAE, 2003; DAMASIO; TRANEL; GRABOWSKI; ADOLPHS; DAMASIO, 2004; SMITH; MEDIN, 1981) que son integrados en una representación lexical para generar una interface con otra información lingüística (VIGLIOCCO; VINSON, 2007). Un tema de central interés es, por lo tanto, conocer cuáles son los rasgos comunes entre aquellas palabras de significados semejantes.

En el presente trabajo, se presentan dos métodos de exploración informatizados diseñados por CIMEPB y por el Grupo de Inteligencia Artificial de la UNMdP, que permiten capturar y analizar información semántica. El primero de ellos, Natural Finder, está destinado al análisis de la vinculación entre palabras a partir de la producción de un individuo y responde en mayor medida a los problemas teóricos de los modelos holísticos. El segundo, Definition Finder permite recolectar los atributos que definen a un concepto para un conjunto de personas y establecer comparaciones entre conceptos y es más pertinente para el estudio de los problemas planteados por los modelos de rasgos. A continuación se describen ambos métodos y se ilustra las posibilidades de análisis que brindan mediante su aplicación a casos concretos.

\section{NATURAL FINDER}

El Natural Finder es un procedimiento recursivo concebido para capturar a partir de un concepto disparador, la red semántica natural de una persona en torno a dicho concepto en ese momento. Este método permite capturar los conceptos que conforman la red semántica próxima del concepto blanco para un individuo. El procedimiento consiste en preguntarle a la persona cuáles son las palabras que asocia con determinado concepto blanco y luego, de modo aleatorio y en forma recursiva, tomar cada una de las palabras producidas como palabra blanco para volver a solicita palabras asociadas. Como resultado de estas estimaciones se producen inexorables referencias cruzadas de diferente magnitud. 
El programa calcula los pesos correspondientes a cada enlace y elabora una matriz cuadrada modo- 1 con las intersecciones de todos los conceptos. Esto permite realizar un análisis de cluster basado en distancias (JOHNSON; 1967) y visualizar una salida a través de un gráfico de red pudiendo apreciar su distribución y los agrupamientos de los conceptos producidos.

\subsection{Descripción técnica}

Ambos sistemas informáticos, el Natural Finder y el Definition Finder han sido implementados en Dolphin (TM), una versión comercial moderna de Smalltalk específica para el entorno Windows (TM). Smalltalk es un lenguaje de programación orientada a objetos puro (GOLDBERG; ROBSON, 1989), diseñado en los laboratorios de investigación de Xerox PARC (Palo Alto Research Center), por el equipo de investigación a cargo del Dr. Alan Kay (SPROULL, 2010). La elección de esta plataforma estuvo fuertemente influenciada por el hecho que Smalltalk presenta diversas ventajas con respecto a otros lenguajes de programación de propósito general, sobre todo en aspectos tales como una representación más natural y simple del modelo y la posibilidad de modificación y depuración del código en tiempo de ejecución.

El modelo diseñado permite representar no sólo el concepto sino también las relaciones que establece el usuario con otras palabras asociadas a dicho concepto. De esta forma, cada una de las palabras asociadas recibe una valoración que tiene en cuenta no solo el orden que el sujeto establece para cada una de ellas, sino también su frecuencia de aparición.

Una vez establecidas las palabras asociadas para el concepto base, se le presenta al usuario cada ellas como si se tratara de un nuevo concepto y se le solicita que ingrese un nuevo conjunto de palabras asociadas con este último.

Este proceso iterativo permite establecer un conjunto de valores que relacionan no solamente a un conjunto de palabras con un determinado concepto sino a todas las relaciones entre todas ellas, lo que permite configurar una estructura bidimensional, denominada sociomatriz. Posteriormente se aplica a la misma, una transformación para obtener una matriz simétrica con la finalidad de simplificar el análisis posterior de los resultados. 
Figura 1. Pantallas de Definición del concepto y consignas

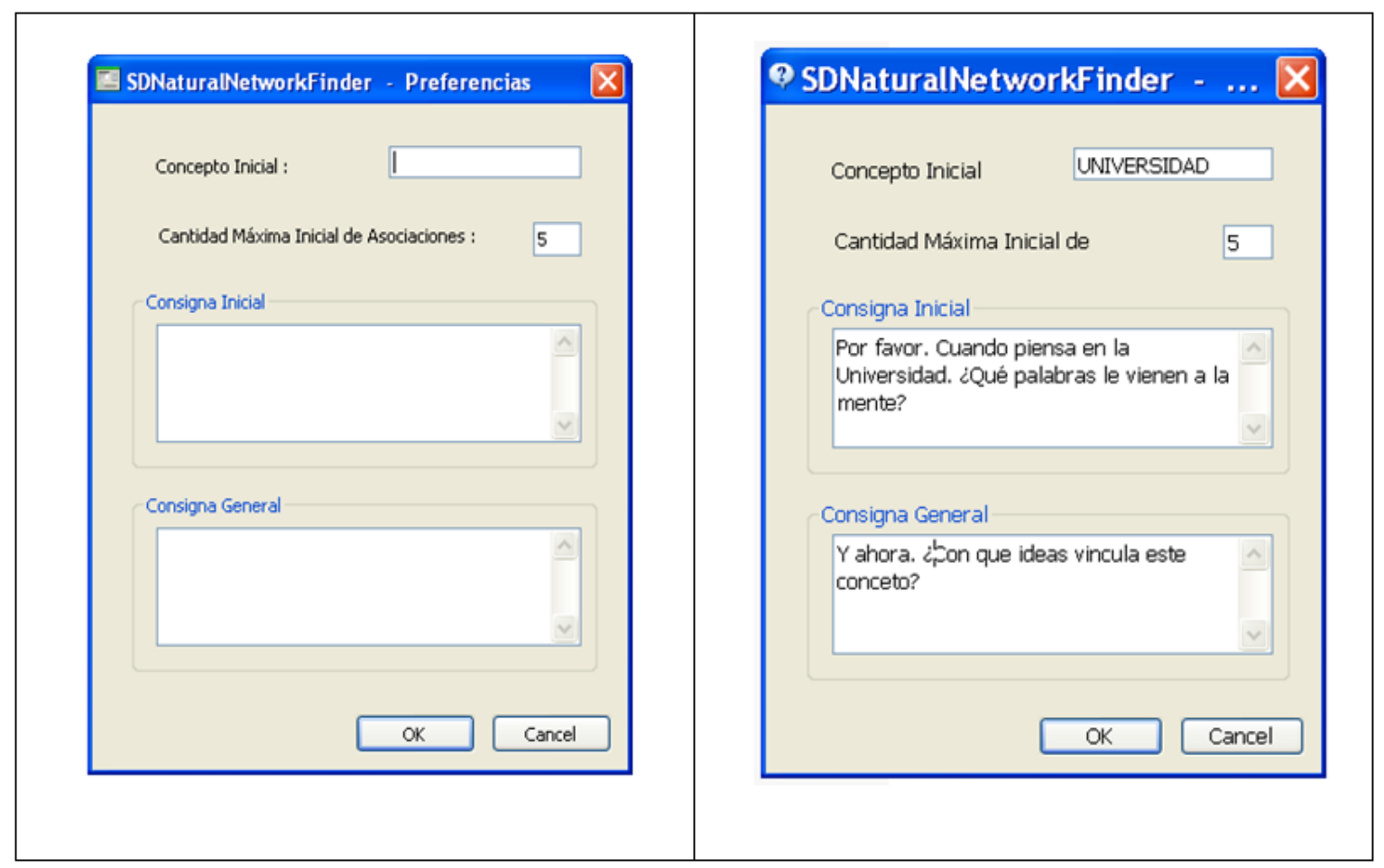

En las Figuras 2 y 3 se muestran cómo le aparece al entrevistado la pantalla inicial y las sucesivas, de acuerdo con las palabras dichas por la persona.

Figura 2. Pantalla tal como le aparece al entrevistado con la consigna inicial

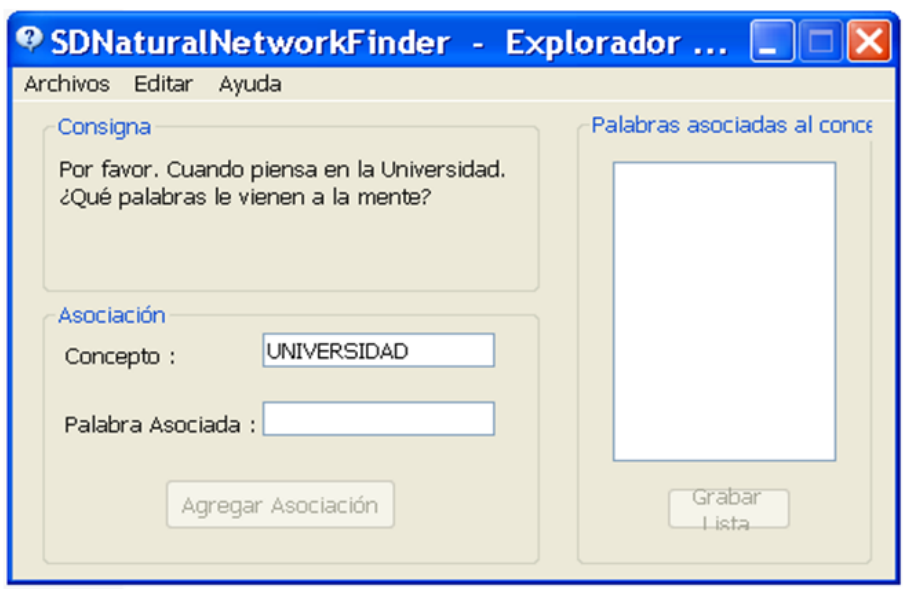


Figura 3. Pantalla recursiva para cada palabra inicial asociada

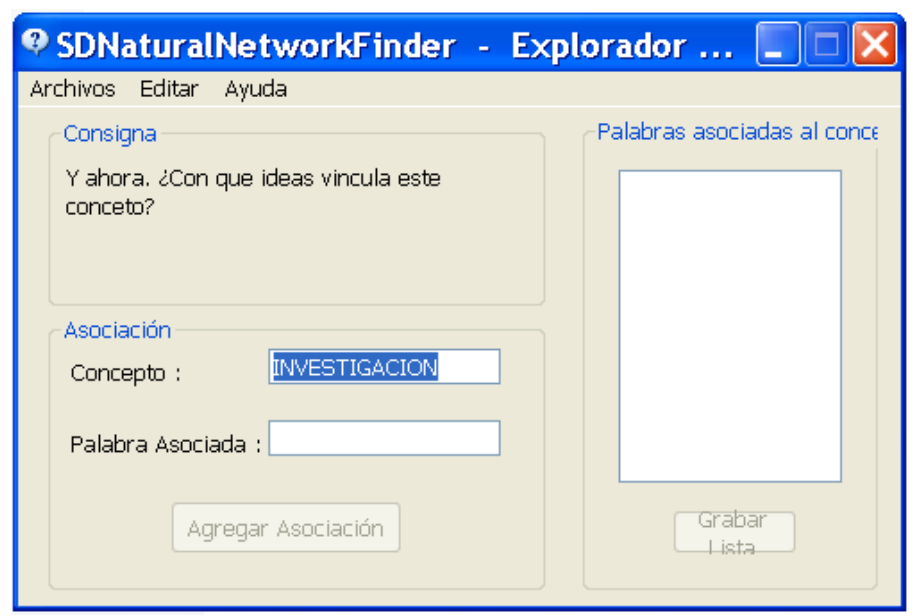

Finalmente, la figura 4 muestra la matriz cuadrada que genera el programa luego de calcular el peso relativo de todas las referencias cruzadas. Para realizar este cálculo inciden en forma diferencial si la referencia de uno a otro concepto incluye su referencia inversa y el orden relativo en el cual se haya producido la referencia. Por ejemplo, no posee el mismo peso si A refiere a B y B refiere a $A$, ambos en primer lugar, que si A se refiere a B en 3er lugar y B se refiere a A en quinto lugar.

Figura 4. Matriz cuadrada disponible para su tratamiento en Análisis de Redes Sociales

\begin{tabular}{|c|c|c|c|c|c|c|c|c|c|c|c|c|c|c|c|}
\hline \multicolumn{16}{|c|}{ E Ejemplo UNIVERSIDAD - Bloc de notas } \\
\hline \multicolumn{16}{|c|}{ Archivo Edición Formato Ver Ayuda } \\
\hline \multirow{2}{*}{\multicolumn{2}{|c|}{$\begin{array}{l}\text { I. 'UNIVERSIDAD' } \\
\text { 'COMUNI }\end{array}$}} & \multicolumn{2}{|c|}{ 'PROGRESO' } & \multirow{2}{*}{\multicolumn{2}{|c|}{ 'SOCIEDAD' }} & \multicolumn{4}{|c|}{ 'INVESTIGACION ' 'ENSEENAAZA' } & \multicolumn{2}{|c|}{ 'CONOCIMIENTO } & \multirow{2}{*}{\multicolumn{2}{|c|}{ 'GESTION' }} & \multicolumn{2}{|c|}{ 'ESTADO' } \\
\hline & & IDAD' & 'PROFE: & SIONAL' & & $A^{\prime}$ & 'TECN & LOGIA' & ENSE & ANZA & POLI & & & & \\
\hline ' UNIVER SIDAD' & 0000 & 0600 & 0400 & 0700 & 0900 & 1000 & 0500 & 0200 & 0100 & 0800 & 0300 & 0000 & 0000 & 0000 & 0000 \\
\hline 'PROGRESO' & 0600 & 0000 & 0000 & 0800 & 0000 & 1000 & 0000 & 0000 & 1333 & 0000 & 0400 & 0000 & 0667 & 0333 & 0000 \\
\hline SOCIEDAD ${ }^{\prime}$ & 0400 & 0000 & 0000 & 0000 & 0000 & 0000 & 0867 & 2000 & 0000 & 1800 & 1000 & 0000 & 0000 & 0600 & 0400 \\
\hline$\therefore N V E D I G A C I O N$ & 0700 & 0800 & 0000 & 0000 & 0250 & 0200 & 0000 & 0000 & 1433 & 0000 & 0000 & 1000 & 0400 & 0000 & 0000 \\
\hline 'ENSENAAZA' & 0900 & 0000 & 0000 & 0250 & 0000 & 0500 & 0000 & 0750 & 0000 & 1000 & 0000 & 0000 & 0000 & 0000 & 0000 \\
\hline ' 'CONOCIMIENTO' & $\begin{array}{l}1000 \\
0500\end{array}$ & $\begin{array}{l}1000 \\
0000\end{array}$ & $\begin{array}{l}0000 \\
0867\end{array}$ & $\begin{array}{l}0200 \\
0000\end{array}$ & $\begin{array}{l}0500 \\
0000\end{array}$ & 0000 & $\begin{array}{r}0000 \\
0000\end{array}$ & 0000 & 1417 & 0000 & $\begin{array}{l}0000 \\
0000\end{array}$ & $\begin{array}{l}0000 \\
0000\end{array}$ & $\begin{array}{r}0500 \\
0000\end{array}$ & $\begin{array}{l}0250 \\
0000\end{array}$ & 0000 \\
\hline 'ESTADO' & 0200 & 0000 & 2000 & 0000 & 0750 & 0000 & 0833 & 0000 & 0000 & 1500 & 0200 & 0000 & 0000 & 0000 & 0250 \\
\hline 'DFGARROI IO' & $\begin{array}{l}0100 \\
0800\end{array}$ & $\begin{array}{l}1222 \\
0000\end{array}$ & $\begin{array}{l}0000 \\
1800\end{array}$ & $\begin{array}{l}1422 \\
0000\end{array}$ & 0000 & $\begin{array}{l}1417 \\
0000\end{array}$ & $\begin{array}{l}0000 \\
0000\end{array}$ & $\begin{array}{l}0000 \\
1500\end{array}$ & $\begin{array}{l}0000 \\
0250\end{array}$ & $\begin{array}{l}0>50 \\
0000\end{array}$ & $\begin{array}{l}0600 \\
0000\end{array}$ & $\begin{array}{l}1000 \\
0000\end{array}$ & $\begin{array}{l}0167 \\
0000\end{array}$ & $\begin{array}{l}0500 \\
0500\end{array}$ & 0000 \\
\hline 'PROFES IONAL' & 0300 & 0400 & 1000 & 0000 & 0000 & 0000 & 0000 & 0200 & 0600 & 0000 & 0000 & 0000 & 0000 & 0800 & 0000 \\
\hline $\begin{array}{l}\text { 'CIINNCIA } \\
\text { 'TECNOLOGIA' }\end{array}$ & $\begin{array}{l}0000 \\
0000\end{array}$ & $\begin{array}{l}0000 \\
0667\end{array}$ & $\begin{array}{l}0000 \\
0000\end{array}$ & $\begin{array}{l}1000 \\
0400\end{array}$ & $\begin{array}{l}0000 \\
0000\end{array}$ & $\begin{array}{l}0000 \\
0500\end{array}$ & $\begin{array}{l}0000 \\
0000\end{array}$ & $\begin{array}{l}0000 \\
0000\end{array}$ & $\begin{array}{l}1000 \\
0167\end{array}$ & 0000 & $\begin{array}{l}0000 \\
0000\end{array}$ & $\begin{array}{l}0000 \\
0000\end{array}$ & $\begin{array}{l}0000 \\
0000\end{array}$ & $\begin{array}{l}0000 \\
0000\end{array}$ & 0000 \\
\hline 'ENSENAANZA' & 0000 & 0333 & 0600 & 0000 & 0000 & 0250 & 0000 & 0000 & 0500 & 0500 & 0800 & 0000 & 0000 & 0000 & 0000 \\
\hline$I C A^{\prime}$ & & & & & & & & & & & & & & & \\
\hline
\end{tabular}


Con esta matriz es posible realizar un análisis de las palabras que se vinculan entre sí con mayor fortaleza, constituyendo una buena representación de un esquema conceptual referido a determinado tema. Para ello se aplica Análisis de Cluster Jerárquico a la matriz en base al procedimiento propuesto por JOHNSON'S (1967). Este método parte de una matriz simétrica $n \times n$ que representa las similitudes o diferencias en un conjunto de $n$ elementos. Lo que el algoritmo encuentra es una serie de particiones anidadas de ítems. Las diferentes particiones se ordenan de acuerdo al aumento (o disminución) de los niveles de similitud (o disimilitud). El algoritmo comienza con la partición de identidad (en el que todos los ítems están en agrupamientos diferentes). Luego junta los ítem que resulten más similares (menos diferentes), para luego considerarlos una sola entidad. El algoritmo continúa recursivamente hasta que todos los ítems se han unido en un solo grupo (la partición completa). De este modo se generan los agrupamientos por mayor cohesión (menor distancia) entre subgrupos y su relación con la totalidad. Para visualizarlo se puede utilizar un software que realice un Escalamiento Multidimensional para reducir las dimensiones antes de graficarlo como por ejemplo el NetDraw (BORGATTI, 2002) o equivalente.

\section{EJEMPLO DE LA UTILIZACIÓN DEL MÉTODO APLICADO A UN DOCENTE UNIVERSITARIO}

Se presentan a continuación, dos ejemplos de la aplicación del Natural Finder a dos personas utilizando como disparador la palabra UNIVERSIDAD.

La Figura 5 muestra los resultados del procesamiento producido sobre las respuestas dadas por una persona adulta mayor, con una gran experiencia en gestión universitaria y funcionario de organismos internacionales.

Se puede visualizar que el procedimiento extrajo cuatro clusters, que para facilitar su exposición hemos redondeado y señalado con las letras A, B, C y D. Su lectura indica la presencia y activación de los siguientes subgrupos de conceptos (esquemas): 
- $\quad$ El cluster A está compuesto por UNIVERSIDAD, TEORÍA, INVESTIGACIÓN, CALIDAD, PROYECTOS y DESARROLLO; este cluster podría ser interpretado como el componente básico de la función investigación propia de las universidades y la inclusión del concepto disparador sugiere la fortísima relación que establece el entrevistado entre la idea de Universidad y el desarrollo de la Investigación. Es interesante destacar que el cluster D vincula fuertemente FINANCIAMIENTO y CALIDAD;

- En el cluster B, compuesto de CONOCIMIENTO, ENSEÑANZA, EVALUACIÓN, PROFESORES, ESTUDIANTES, GRUPOS, PRÁCTICAS, PROGRAMAS, APRENDIZAJE y CLASES, parece visualizarse con claridad la función docente de la universidad. Cabe señalar aquí la presencia del cluster $\mathrm{C}$, que señala la fuerte vinculación entre FORMACIÓN y COMPETENCIA y la EVALUACIÓN de los PROFESORES (si se observa con detenimiento se pueden apreciar lazos fuera del cluster que sugieren la relación entre EVALUACIÓN y CALIDAD).

En síntesis, la interpretación de la figura resultante parece indicar que, cuando el entrevistado se encontró con la palabra UNIVERSIDAD activó un esquema general que muestra a la universidad como constituida por dos grandes componentes, investigación y docencia. La primera se asocia con la generación de proyectos y teorías, y su calidad se encuentra asociada a los recursos financieros que se hallen disponibles para sustentarla. Mientras que la docencia, sobre todo en su aspecto referido a la calidad, parece estar vinculada con la formación y competencia de sus docentes. 


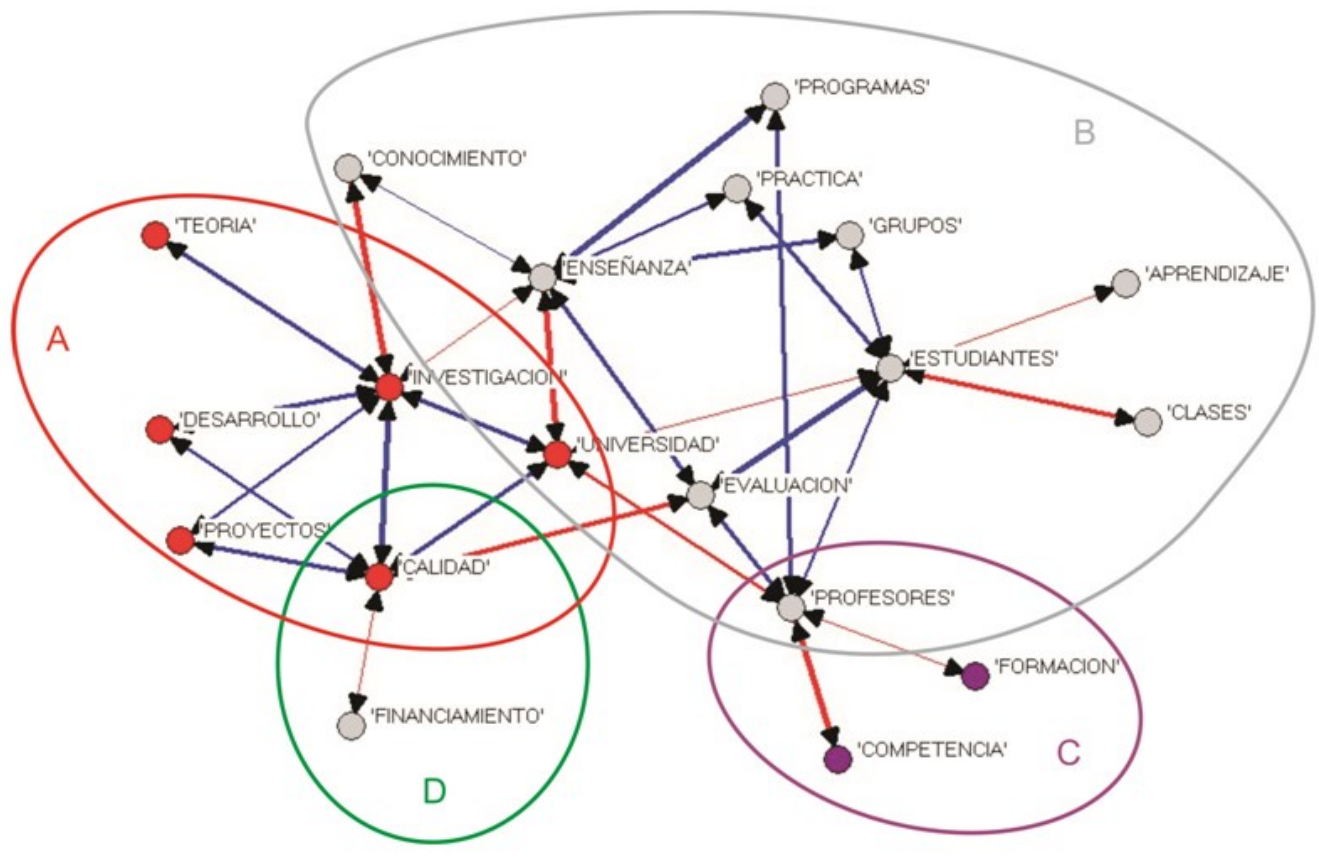

La Figura 6 que, se presenta a continuación, muestra los resultados del procesamiento producido sobre las respuestas dadas por un docente universitario especializado en Educación y con una gran experiencia en el área de ingreso a la Universidad.

Se puede visualizar que el procedimiento extrajo tres clusters que, para facilitar su exposición hemos redondeado y señalado con las letras A, B y C. Su lectura indica la presencia y activación de los siguientes subgrupos de conceptos (esquemas):

- $\quad$ El cluster A está compuesto por CAPITAL CULTURAL, ORGANIZADORES SEMÁNTICOS, ESTRUCTURA SEMÁNTICA, ORGANIZADORES SINTÁCTICOS, COMPRENSIÓN TEXTUAL Y CONOCIMIENTO PREVIO; este cluster podría ser interpretado como las actividades básicas requeridas a los alumnos para producir la comprensión de contenidos novedosos remitiendo, por lo tanto, al aspecto de la función docente de la Universidad;

- El cluster B está compuesto por UNIVERSIDAD, ESCRITURA Y ALFABETIZCIÓN ACADÉMICA siendo que, estos dos últimos, se vinculan fuertemente dentro del cluster con COHESIÓN, COHERENCIA y LINGÜÍSTICA, 
por un lado, y REGLAS DE TRANSMISIÓN, NOVATO, CAMPO DISCIPLINAR, EXPERTO Y REGLAS DE PRODUCCIÓN, por el otro. La perplejidad hermenéutica inicial que seguramente se produce en el lector comienza a disiparse cuando advertimos que también el cluster B se refiere a las actividades del alumno vinculadas con sus aprendizajes; sólo que en esta oportunidad los alumnos a los que hace referencia, a diferencia de los mencionados en el cluster $\mathrm{A}$, son los ingresantes a la Universidad, aquellos novatos a quienes hay que alfabetizar académicamente. Es interesante señalar aquí que la única razón de la separación de los cluster A y B, ya que ambos se refieren a la docencia, es que los destinatarios de esta docencia son, o bien alumnos ingresantes o bien alumnos avanzados en la carrera;

- $\quad$ El cluster C permite una lectura más directa y evidente. Se compone de AUTONOMÍA y EDUCACIÓN SUPERIOR, ambas vinculadas al COGOBIERNO, lo que implica LEYES PROPIAS y LIBRE ELECCIÓN. De este modo, parecería que el cluster C considera la forma en que la Universidad pública gestiona la administración de sus recursos. A su vez, aparece DISCIPLINAS CIENTÍFICAS vinculado con EDUCACIÓN SUPERIOR pero, sin vinculación con los otros elementos del cluster. Esto podría interpretarse como que la educación superior está compuesta por disciplinas científicas y su autonomía se manifiesta a través del co-gobierno, la libre elección y las leyes propias. 


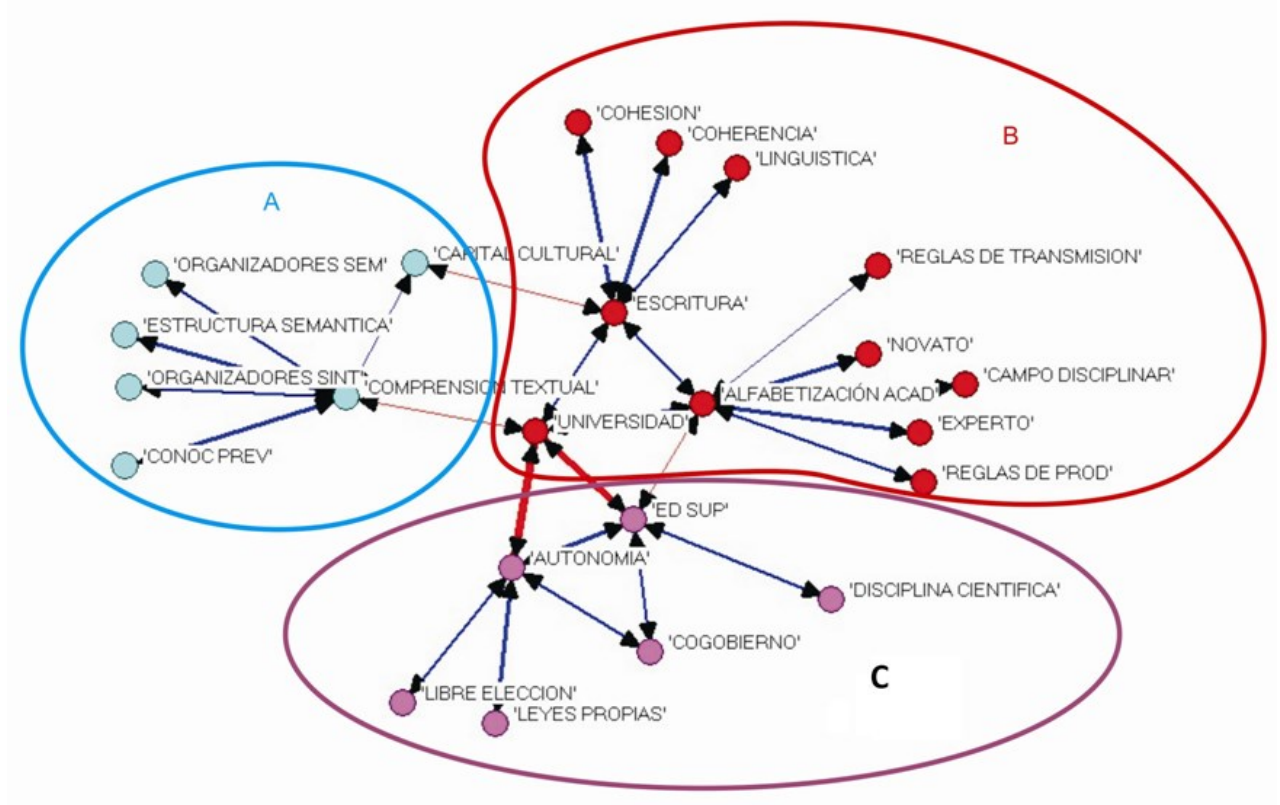

En resumen, el grafo muestra que, en la primera representación evocada por este docente al aparecer el término UNIVERSIDAD, se está refiriendo a su función docente y a su estructura de gobierno. A pesar de que son harto conocidas por el docente las funciones de investigación, desarrollo y transferencia, no se activaron las representaciones correspondientes 0 , al menos, no alcanzaron a producir la alocución de la etiqueta verbal correspondiente.

\subsection{Definition finder}

En el estudio de la memoria semántica, es de particular interés conocer cuáles son los atributos que tienen mayor importancia en la definición de un concepto, dado que las cualidades de los atributos y sus relaciones son una variable fundamental en la estructura interna de un concepto (MOSS et. al. 2007). Hay atributos que son más centrales y otros más periféricos. Esto ha sido estudiado mediante diversos constructos como la distintividad (DEVLIN; GONNERMAN; ANDERSEN; SEIDENBERG, 1998) y la relevancia (SARTORI; LOMBARDI, 2004). El Definition Finder es un programa informatizado que aporta un dato de interés en este sentido ya que permite capturar el conjunto de 
atributos que definen a un concepto blanco de manera ponderada. Es decir que, arroja una lista de los atributos que definen a un concepto ordenándolos por el peso decreciente. A diferencia del Natural Finder, este programa permite calcular el grado de comunalidad de los atributos de un concepto para una comunidad lingüística determinada. Se parte de una tarea de producción de atributos semánticos donde las personas deben enunciar los atributos que a su entender mejor definen a cierto concepto. Como resultado de este tipo de tareas se obtienen dos clases de información: la frecuencia de aparición de cada atributo y el orden en que aparecen. El Definition Finder permite conjugar ambos y obtener el dato del peso ponderado para cada atributo. Los resultados toman valores numéricos de entre 0 y 1 , siendo 1 el mayor peso. A su vez, es posible graficar la secuencia de atributos definidores mediante una curva ordenada por peso decreciente de cada atributo para un concepto.

\subsection{Descripción técnica}

La metodología aplicada en el desarrollo de ambos sistemas informáticos (Natural Finder y Definition Finder) es la denominada Test Driven Development (BECK, 2003). Esta metodología consiste en el diseño de un conjunto de pruebas de comportamiento del software a desarrollar, a medida que se va implementando el sistema mismo. Esto permite una detección muy temprana de errores de implementación, lo que conduce a una disminución de los tiempos de implementación y constituye un mecanismo de seguridad adicional en el momento de incorporar nuevas funcionalidades. Para su aplicación se utilizó el framework Sunit, presente en Dolphin (TM), y se generaron conjuntos de pruebas automáticas para cada uno de los objetos que forman parte del modelo.

El modelo informático del Definition Finder utiliza una técnica similar a la descripta anteriormente, aunque en este caso, cada conjunto de definidoras es ingresado por un usuario diferente. De esta forma, cada usuario establece un conjunto de definidoras o palabras asociadas a un determinado concepto. A partir de estas respuestas es posible calcular una valoración para cada una de ellas teniendo en cuenta el orden y la frecuencia de aparición, reiterando este proceso con cada integrante del grupo. 
Además, como los valores obtenidos están basados en el orden y en la frecuencia de aparición, se hace necesario normalizar dichos valores con el objetivo de poder comparar los resultados obtenidos con diversos grupos, en los que la cantidad de integrantes puede ser diferente.

\subsection{Ejemplo del Definition Finder aplicado a dos conceptos de la categoría} frutas

Para ilustrar la aplicación del método se presenta a continuación, un ejemplo de su utilización para los conceptos TOMATE y LECHUGA. Los datos fueron obtenidos de las Normas de Producción de Atributos Semánticos para castellano rioplatense (VIVAS et. al., 2011). La muestra estuvo conformada por 30 personas adultas universitarias.

El procedimiento de carga y procesamiento se desenvolvió como muestra la Figura 7.

Figura 7. Proceso de carga y procesamiento de atributos.

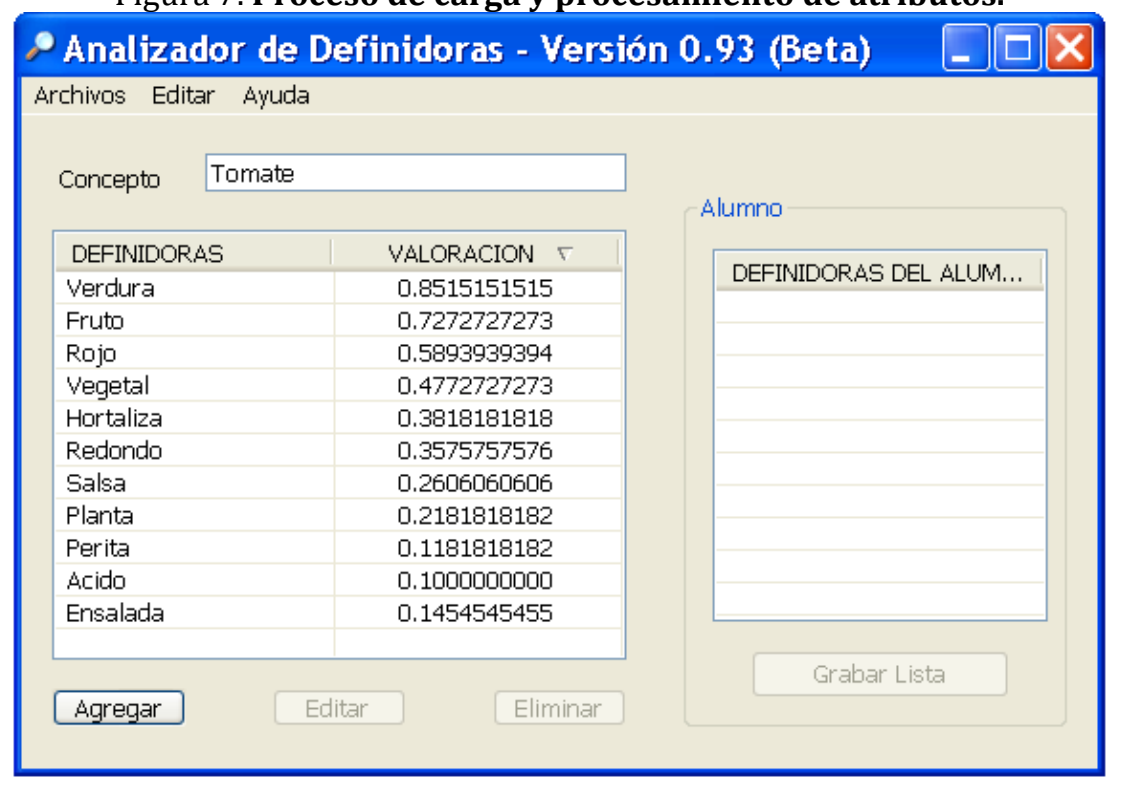

La salida resultante de la carga de los datos en el Definition Finder para cada concepto se puede observar en las Tablas 1 y 2 . 
Tabla 1. Salida del Definition Finder para el concepto 'tomate’

\begin{tabular}{|l|l|}
\hline \multicolumn{1}{|c|}{ DEFINIDORAS } & VALORACIÓN \\
\hline verdura & 0.966 \\
\hline fruto & 0.875 \\
\hline vegetal & 0.866 \\
\hline rojo & 0.852 \\
\hline hortaliza & 0.733 \\
\hline redondo & 0.65 \\
\hline salsa & 0.533 \\
\hline planta & 0.533 \\
\hline perita & 0.5 \\
\hline acido & 0.5 \\
\hline ensalada & 0.466 \\
\hline
\end{tabular}

Tabla 2. Salida del Definition Finder para el concepto 'lechuga'

\begin{tabular}{|l|l|}
\hline DEFINIDORAS & VALORACIÓN \\
\hline verdura & 0.94 \\
\hline vegetal & 0.795 \\
\hline verde & 0.718 \\
\hline planta & 0.6 \\
\hline hojas & 0.588 \\
\hline ensalada & 0.545 \\
\hline comida & 0.475 \\
\hline tallo & 0.466 \\
\hline fresca & 0.45 \\
\hline sana & 0.4 \\
\hline arrugada & 0.375 \\
\hline
\end{tabular}

En la columna a la izquierda de cada tabla, aparecen los atributos comunes dados por este grupo para cada concepto y en la columna derecha de las tablas la valoración (pero relativo) de cada uno de los atributos comunes dados por este grupo y, en función de este peso, se les asigna el orden. A mayor peso mejor ubicación en la lista.

$\mathrm{Al}$ analizar los atributos dados por las personas para ambos conceptos, se puede observar que presentan algunos atributos comunes que tienen un peso muy alto (verdura y vegetal) y otros que los diferencian (ej., rojo y verde). Los atributos comunes se refieren a la categoría semántica supraordinada que indica la clase de objetos a la cual pertenecen, mientras que los no compartidos describen los atributos o características particulares de cada concepto. A su vez, es interesante ver que primero se produce la categoría semántica de pertenencia y luego aparecen atributos relevantes en la definición del concepto, 
apareciendo más adelante atributos menos específicos, como ÁCIDO o ENSALADA.

\section{DISCUSIÓN}

Este trabajo tuvo como objetivo principal mostrar dos métodos informatizados de fácil administración para extraer y analizar una red semántica tanto individual como grupal. Ambos métodos permiten conocer cómo se representan los significados de las palabras y comparar varias redes pertenecientes a diferentes individuos o grupos. Tal como se ilustró, el Natural Finder resulta de utilidad para el análisis de la vinculación entre palabras a partir de la producción de un individuo y responde en mayor medida a los problemas teóricos de los modelos holísticos (COLLINS; LOFTUS, 1975; DE ALMEIDA, 1999). Como pudo observarse en los ejemplos presentados, las salidas gráficas de este método muestran la representación de la red semántica vinculada con el o los conceptos dados y, a su vez, permite analizar las relaciones de todos los conceptos entre sí que fueron dados en las sucesivas asociaciones, como así también, observar la conformación de cluster o esquemas entre dichos conceptos.

Como se puede advertir, el Natural finder ha sido diseñado con el propósito de visualizar y comprender los esquemas y subequemas de conocimientos y creencias involucrados en una situación particular para un sujeto individual. Dadas estas características, su campo de aplicación se orientó a utilizaciones clínicas, promoviendo investigaciones en el ámbito de la salud, en el estudio de los mapas semánticos del dolor en pacientes con implante dental (LAMAS, 2014), en el desarrollo de modelos de prevención del riesgo suicida, entre otros.

El Definition Finder, por su parte, permite recolectar los atributos que definen a un concepto para un conjunto de personas integrantes de una misma comunidad lingüística y establecer comparaciones entre los diferentes tipos de conceptos $\mathrm{y}$, entre las diferentes comunidades lingüísticas. Como ya se mencionó, este programa es más pertinente para el estudio de los problemas planteados por los modelos de rasgos (SMITH; MEDIN, 1981; CREE; MCRAE, 
2003) уa que, la información que arroja permite visualizar el conjunto de atributos que definen a un concepto considerando el peso relativo de cada uno, como así también, comparar a los conceptos entre sí. Esta aplicación se utilizó recientemente con los atributos extraídos de las Normas de Producción de Atributos Semánticos para Castellano Rioplatense (VIVAS; COMESAÑA; GARCÍA CONI; VIVAS; YERRO, 2011) para otorgarles un peso diferencial a los atributos, tal que permita la medición de las distancias semánticas entre diferentes conceptos. También se utilizó para establecer el criterio del orden de presentación de los atributos en tareas de reconocimiento de conceptos referidos a emociones básicas (GARCIA; PAGNOTTA; PAZGON; VIVAS, 2013) y objetos concretos (VIVAS; MACINTYRE; VIVAS, RICCI, no publicado).

Ambas herramientas son de fácil utilización y presentan una interfaz de usuario amigable. Un aspecto adicional a destacar es que ambos métodos son de libre disposición y pueden ser descargados de manera gratuita desde el sitio labpsi.mdp.edu.ar.

\section{REFERENCIAS}

BECK, K. Test Driven Development. 1ra. Ed., pp 240, Editorial Addison Wesley Professional. 2003.

BORGATTI, S. NetDraw. Natick: Analytic Technologies. V 0.60. 2002.

COLLINS, A. M.; LOFTUS, E. F. A spreading-activation theory of semantic processing. Psychological Review. 1975, vol. 82, p. 407-428.

CREE, G. S. y MCRAE, K. Analyzing the factors underlying the structure and computation of the meaning of chipmunk, cherry, chisel, cheese, and cello (and many other such concrete nouns). Journal of Experimental Psychology: General. 2003 , vol. $132,163-201$.

DE ALMEIDA, R.G. What Do Category-Specific Semantic Deficits Tell Us about the Representation of Lexical Concepts? Brain and Language. 1999, 68, 241-248.

DAMASIO, H., TRANEL, D., GRABOWSKI, T., ADOLPHS, R., DAMASIO, A. Neural systems behind word and concept retrieval. Cognition, 2004, 92, 179-229. doi:10.1016/j.cognition.2002.07.001

DEVLIN, J.T., GONNERMAN, L.M., ANDERSEN, E.S., SEIDENBERG, M.S. Category specific semantic deficits in focal and widespread brain damage: A 
computational account. Journal of Cognitive Neuroscience. 1998, vol. 10, p. 7794.

GARCIA, PAGNOTTA, L., PAZGON, E., VIVAS, J. Poder de discriminación de los atributos semánticos. Mínima cantidad de descriptores requeridos para identificar conceptos emocionales. En V. Jaichenco y Y. Sevilla (Coords.), Psicolingüística en español. Homenaje a Juan Seguí. Bs. As.: Editorial FFyL UBA., 2013, p. 95-109.

GOLDBERG, A., ROBSON D. Smalltalk-80 The Language. 1ra. Ed., pp 608, Editorial Addison Wesley Publishing Co. 1989.

JOHNSON, S. C. Hierarchical Clustering Schemes. Psychometrika. 1967, vol. 2, p. 241-254.

KAHNEMAN, D.; TVERSKY, A. On the psychology of prediction. Psychology Review. 1973, vol. 80, p. 237-251.

LAMAS, V. “Representación semántica del implante dentario, Ansiedad y Percepción Psicológica del dolor postoperatorio en pacientes sometidos a cirugía de implante oral”. Director: Dr. Jorge Vivas. Trabajo para obtener el grado de Lic. en Psicología, Facultad de Psicología. UNMDP, 2014.

MCNAMARA, T. P.; HOLBROOK, J. B. Semantic Memory and Priming. En: A. F. HEALY; R. W. PROCTOR (Eds.) Handbook of Psychology Volume 4 Experimental Psychology. New Jersey: John Wiley \& Sons, Inc., 2003, p. 447-474.

MOSS, H.E.; TYLER, L.K.; TAYLOR, K.I. Conceptual structure. En: G. GASKELL (Ed.), Oxford Handbook of Psycholinguistics. Oxford, UK: Oxford University Press, 2007, p. 217-234.

SARTORI, G., LOMBARDI, L. Semantic relevance and semantic disorders. Journal of Cognitive Neuroscience, 2004, 16, 439-452. Doi:

10.1162/089892904322926773

SMITH, E.E., MEDIN, D.L. Categories and concepts. Cambridge, MA: Harvard University Press, 1981.

SPROULL B. Alan Kay a visionary designer, Points of View: a tribute to Alan Kay. 1ra Ed., Editorial Typecraft Wood \& Jones Inc., pp 1-4, Pasadena, California, USA. 2010.

VIGLIOCCO, G., VINSON, D.P. Semantic representations. En: G. Gaskell (Ed.), The Oxford Handbook of psycholinguistics. Oxford, UK: Oxford University Press, 2007. p. 195-215.

VIVAS, L., MACINTYRE, M., VIVAS, J. Y RICCI, L. Variables psicolingüísticas involucradas en la evocación de conceptos a partir de la presentación de atributos sucesivos. Manuscrito no publicado. 
VIVAS, J., COMESAÑA, A., GARCÍA CONI, A., VIVAS, L., YERRO, M. Distribución de los atributos semánticos en función del tipo de categoría y campo semántico. Resultados preliminares para la confección de normas de atributos. En: M.C. Richaud y V. Lemos (comp.) Psicología y otras ciencias del comportamiento. Compendio de investigaciones actuales. Tomo 1. Libertador San Martin: Editorial Universidad Adventista del Plata, 2011, p.311-333. 